\title{
Envisioning Molecular Network of Obesity by Omics Stratification
}

\author{
Bartley $\mathrm{S}^{1}$, Cho $\mathrm{HH}^{2}$, Cho $\mathrm{JH}^{\star 2}$, and Kim $\mathrm{HD}^{\star 1}$
}

${ }^{1}$ Department of Public Health, Division of Environmental Health Science, New York Medical College, Valhalla, NY, USA

${ }^{2}$ Institute of Animal Medicine, College of Veterinary Medicine, Gyeongsang National University, Jinju, Korea

*Corresponding author: Kim HD Ph.D, Department of Public Health, Division of Environmental Health Science, New York Medical College, Valhalla NY 10595, USA, Fax: +1-914-594-4576, Tel: +1-914-594-4259, E-mail: hongduck_kim@nymc.edu

Cho JH, D.V.M. Ph.D, Jiju Daero 501, Jinju-city, Gyeongsangnamdo, 660-701, Korea, College of Veterinary Medicine, Gyeongsang National University, Fax: +82-55-772-2349, Tel: +82-55-772-2358, E-mail: jaehcho@ gnu.ac.kr

Citation: Bartley S, Cho HH, Cho JH, Kim HD (2018) Envisioning Molecular Network of Obesity by Omics Stratification. Nutr Health Sci 5(2): 201. doi: 10.15744/2393-9060.5.201

Received Date: February 06, 2018 Accepted Date: April 03, 2018 Published Date: April 12, 2018

\begin{abstract}
To date, Obesity is considered prime concern of public health with multi-dimensional factors engaged in metabolic discrepancy that is rooted from multifactorial causes (i.e., environmental intoxication or genetic abnormality), and the unmet landscape of health care system seeks for therapeutic or preventive measures. Compelling evidence support Omics as strategic tool of systemic biology is benefit to define wiring of molecular circuit in metabolic malfunction and evaluation of metabolic modulator such as synthetic drugs, stem cell replacement, and natural secondary metabolites from various resources.

The interface between the nutritional environment and cellular/genetic processes is referred to as Nutritional genomics or Nutrigenomics. It seeks to understand the effects of diet on an individual's genes and health. Nutrigenomics seeks to provide a genetic understanding for how common dietary chemicals (i.e., nutrition) affect the balance between health and disease by altering the expression or structure of an individual's genetic makeup. In addition, a prevention thread in public health informatics still demand to improve detection skills from patient's clinical samples and monitoring disease development surrounding reserve population who may have more exposure risk to various micro- and /or macro-environmental determinants in disease susceptibility. Molecular-based detection system can predict evolving disease with respect to various modification of key molecule which might attribute to pathogenesis of disease that reflect heredity including quantitative trait loci, polymorphism, and epigenetic modification utilize omics platform including epigenetics, metabolomics, nutrigenomics, transcriptomics, pharmacogenomics, and genomics. Moreover, patterned or personalized approach to treat metabolic disease could be utilized by structuring each algorism associated with molecule chain based surveillance and prevention system. To promote effective biomarker discoveries in the field of molecular therapy and early detection target molecule in obesity, innovation tactics and earlier diagnostic tools as potential strategy have given us inspiring in clinical validation and assessment to improve prevention/treatment outcome for better quality in the process of translation medicine.

In this short review, we proposed that OMICS technologies is worthy to develop clinical assessment with regard to diagnose, predict, and treat chain of metabolic diseases (i.e., initiation, progress and determination) and define causative molecules based wiring on host defense machinery after exposure various unknown environmental agents such as harmful bacterial infection or pathogen, heavy metal in air pollution, and environmental stressor as an environmental threat. As a key strategical approach to detect genetic variation or unveil malfunction of host defense system in metabolic disorder, Nutrigenomics can, not only, be used to aid in assessing one's risk for obesity, but it can also be used to treat obesity by establishing personalized diet plans.
\end{abstract}

Keywords: Obesity; Metabolic disorder; Nutrigenomics; Molecular therapy; Genetic variation

\section{Introduction}

Obesity is a growing public health problem. According to WHO, the "worldwide obesity rate has doubled since 1980". Even more alarming, at least 2.8 million people die each year as a result of being overweight or obese [1]. This health crisis has put a financial burden on many nations across the globe. The Centers for Disease Control and Prevention estimates that the United States spent $\$ 147$ billion dollars on medical costs related to obesity in 2008 [2]. It is a costly and very complex issue; therefore, a great deal of research and resources have been allocated to the prevention and treatment of obesity.

Obesity is considered a complicated metabolic disorder that is rooted from multifactorial causes, and the unmet landscape of health care system seek to better surveillance by facing challenging issues about what genomics data gave us with regards to 
resolution with certainty of early detection skill and mitigate an epidemic concern through unfolding genetic or environmental risk factor which may affect their molecular network could derail functional window after exposure to those factors in public health $[3,4]$. Increasing economic burden to manage obesity is highly skew to low income and less educated populations which are more susceptible, vulnerable, and results in high prevalence of disease rate along with concurrent secondary disease occurrence. To date, a prevention thread in public health informatics still demand to improve detection skills from patient's clinical samples and monitoring disease development surrounding reserve population who may have more exposure risk to various micro- and / or macro-environmental determinants in disease susceptibility. Until now, the channel of molecular surveillance program which was designed to capture quality of genomics move forward in the field of innovative therapeutic measures or exploring diagnostic molecular biomarker along with relevant phenotype clinical outcome. At present, compelling evidence indicate the connection between disease pathogenesis and genetic variation in metabolic disease has unveiled their malfunction within metabolic network through deciphering targeting receptor or signaling molecules such as fatty acid oxidation (FAO) gene regulators, PPAR $\gamma$ coactivator (PGC-1 $\alpha$ ), peroxisome proliferator-activated receptors (PPARs), and nuclear respiratory factors (NRFs). This suggests that patterned or personalized approach to the category of metabolic disease could be utilized in molecule based surveillance system by omics platform including epigenetics, metabolomics, nutrigenomics, transcriptomics, pharmacogenomics, and genomics as a potential surveillance module [5]. In the case of diabetic nephropathy, DNA probe-based assessment tools [6] have shown beneficial aspects on how it facilitates to develop design in gene ontology and discovery of metabolic disease process along with visualization use analyze software, validation of targeted gene annotation in human renal tissue. Molecular-based detection system can predict evolving disease with respect to various modification of a molecule which might attribute to pathogenesis of disease that reflect heredity including quantitative trait loci, polymorphism, and epigenetic modification. Many of these diseases and disorders are preventable; a prime example of such a preventable metabolic disease is obesity. According to the World Health Organization, obesity is "defined as abnormal or excessive fat accumulation that may impair health" [1]. The Body Mass Index (BMI) (a calculation that uses one's height and weight) is often used to determine if an individual is overweight or obese. A person with a BMI over 30 is considered obese [1]. Because there are many different causes of obesity, it is a complex disorder. Both an increase in food consumption, particularly of those foods high in fat and calories, and a reduction in physical activity can cause excessive weight gain leading to obesity [1]. Coronary heart disease, stroke, type II diabetes, osteoarthritis and certain cancers are chronic diseases linked to obesity [7]. There are a broad range of risk factors associated to obesity prevalence as following: genetics, family lifestyle, inactivity, unhealthy diet, medical problems, certain medications, socioeconomic status, age, pregnancy, stress, smoking, and lack of sleep [8].

\section{Results}

Numerous clinical-based data mining with regard to molecular abnormality could alter phenotypic variation provided to us through the various disciples of the study of OMICS platform including Proteomics (study of proteins), Metabolomics (study of metabolite), Cellomics (study of cellular signaling), Lipidomes (study of lipids and their interacting partners), and Glycomics (effects of sugars) allows us to better understand the development of disease progression with both internal and external risk factors in the micro- and/or macro-environment, respectively. Certain type of molecular based risk assessment strategies such as detection of gene variation in metabolic disease allows us to prevent risk factor (i.e., exposure of poisoning/harmful agents) that surround our environment which leads to poor health. Sajuthi S. et al. [9] reported that visualized genetic variants (i.e., CHURC1, USMG5, and ERAP2) expressed in both adipose and muscle tissue following analysis of expression quantitative trait loci (eQTLs) and abnormality of metabolic phenotype was measured by gene expression variants likely T2D (e.g., PIK3C2A, RBMS1, UFSP1), gluco-metabolic phenotypes (e.g., INPP5E, SNX17, ERAP2, FN3KRP), and obesity (e.g., POMC, CPEB4) that utilized a genome-wide association study (GWAS) in African ancestry populations. These outcomes have shown genetic variation in regulatory gene expression that could be closely associated with susceptibility to disease development such as type 2 diabetes (T2D), glyco-metabolic phenotypes, and obesity. Similarly, another study conducted by Bureau A. et al. [10] which focused on how environmental exposures impact disease pattern (endophenotypic variation) following affected trait was reported with regard to correlation between metabolic abnormality and genetic variation. It was suggested that a gene trait variation due to exposure to environment factor in the population of Quebec Family is associated with metabolic syndrome such as high level of physical activity without abnormal obesity by detecting positive association of the A allele (rs1375713) in the NOX 3 gene.

Effective biomarker discoveries in the field of molecular therapy and diagnostic have given us inspiring innovation tactics and earlier diagnostic tools as potential strategy in clinical validation and assessment to improve treatment outcome for better quality in translation medicine. The fat mass and obesity-associated gene (FTO) is another genetic variant that is linked to obesity [11]. According to Barh et al. [11], those who carry the A allele of this gene have a higher BMI than individuals who carry the T allele. Their findings also indicate that this variant plays a role in the amount of food one consumes [11]. It still remains to be determined whether the therapeutic value depends on personal medicine likely to be a genetic predisposition between monogenic to polygenic in metabolic disorder such as Obesity prone families. For example, FTO gene which causes increase body mass index was found in T2D (type 2 diabetes mellitus) following GWAS analytic platform with regard to unveil disease etiology, personal health care, and clinical beneficiary [12-14]. Further progression likely polygenic variants were detected in 17 independent genomics regions, and validation cross-link between regulatory gene variants in body weight and heritability of obesity as family trait in human subject has not been determined yet [15]. 


\section{Discussion}

Currently, it appears that the impact of detection tool with respect to patronize genetic modification prone to disease development by applying eminent OMICS technologies have allowed us to gain a more comprehensive understanding of risk factors to define conceptual issue such as gestational programming along with multiple OMICS likely combination of nutrigenomics, metabolomics to detect epigenetic alterations, and/or disease determinant concomitant with quality of environmental factors including air, food safety, and chemical safety. In the previous study, it was indicated that metabolic abnormalities reflect epigenetic modification including alteration of DNA methylation, histone variation, aberrant chromatin remodeling and/or generation of microRNAs due to regulatory alteration in obesity or prenatal obesity was influenced by molecular epigenetic reprogramming along with different risk factors such as nutritional and environmental agent exposures during the pregnancy period [16].

In the future, gene or enzyme-based would be implemented with OMICS technologies to develop clinical bio-sensors to diagnose, predict, and treat metabolic diseases against various unknown harmful biological infection or pathogen as an environmental threat. Overall, the advancement of these OMICS-based tools can help lead many people to have a better quality of life following identification and visualization of genomics trait due to genetic variation or environmental influence.

Nutrigenomic as a domain of validation or evaluation detection tool would be considered a useful assessment platform of OMICS in combating environmental pollution such as food poisoning, overuse of antibiotic in livestock, and soil contamination is a concern of the global obesity epidemic. Nutrigenomics is defined as "the study of the effects of nutrients on the expression of an individual's genetic makeup" [17]. This field of study is multifaceted, using techniques from genomics, proteomics and metabolomics. According to a published report in Obesity Reviews, there are more than 600 genes, markers, and chromosomal regions linked to obesity [18]. The presence, absence, and/or variation of these genetic markers can be used to assess an individual's risk for becoming overweight or obese. For example, a 2003 study found that women with a Glu27 gene variant and high carbohydrate intake (with $49 \%$ of calories consumed coming from carbohydrates) "had a nearly three-fold increase in their risk of developing obesity" [17]. Conversely, women with a different variant of the same gene did not have an increased risk of being obese when consuming the same number of calories from carbohydrates [17]. One can infer from this study that because of the gene variation, two individuals consuming the same diet can result in different weight outcomes.

\section{Conclusion}

As a key strategical approach to detect genetic variation in metabolic disorder, Nutrigenomics can, not only, be used to aid in assessing one's risk for obesity, but it can also be used to treat obesity by establishing personalized diet plans. For instance, researchers developed personalized calorie-controlled diet program for weight reduction using 24 variants in 19 genes [19]. They compared weight loss and maintenance in 50 individuals who received customized exercise and dietary guidelines based on their genotype to 43 individuals who received only generic diet and exercise advice [19]. The study demonstrated that individuals receiving personalized dietary advice based on their genetic makeup were more successful in losing weight and maintaining weight loss than those who did not.

Taken together, disease surveillance as part of the structure of public health informatics can use OMICS-driven technology to prevent, diagnose, and treat numerous disorders includes obesity. Genova and MetaMetrix are just a few companies that sell genomic profiling test to aid in dietary decisions [17]. Although, the obesity epidemic presents a unique challenge to public health officials for many years to come, the use of nutrigenomics offers promising solutions to this growing problem. Genetically inspired, personalized care equipped with nutritional programs via data visualization tool such as nutrigenomics technologies will insure a healthier tomorrow.

\section{Acknowledgement}

This research was supported by Basic Science Research Program through the National Research Foundation of Korea (NRF) funded by the Ministry of Education (No. 2016R1D1A1A09918213).

\section{References}

1. World Health Organization (2016) Obesity and overweight. Geneva, Switzerland.

2. Centers for Disease Control and Prevention (2016) Overweight and obesity: adult obesity facts.

3. Ravussin E, Bogardus C (2000) Energy balance and weight regulation: genetics versus environment. Br J Nutr 83: 17-20.

4. Speakman JR (2004) Obesity: the integrated roles of environment and genetics. J Nutr 134: 2090-105S.

5. Maples JM, Brault JJ, Shewchuk BM, Witczak CA, Zou K, et al. (2015) Lipid exposure elicits differential responses in gene expression and DNA methylation in primary human skeletal muscle cells from severely obese women. Physiol Genomics 47: 139-46.

6. Cohen CD, Lindenmeyer MT, Eichinger F, Hahn A, Seifert M, et al. (2008) Improved elucidation of biological processes linked to diabetic nephropathy by single probe-based microarray data analysis. PLoS One 3: e2937.

7. Wolf AM (2001) The economic impact of obesity. Building bridges with managed care. Postgrad Med 109: 34-9.

8. Mayo Clinic (2016) Obesity: risk factors.

9. Sajuthi SP, Sharma NK, Chou JW, Palmer ND, McWilliams DR, et al. (2016) Mapping adipose and muscle tissue expression quantitative trait loci in African Americans to identify genes for type 2 diabetes and obesity. Hum Genet. 135:869-80. 
10. Bureau A, Croteau J, Couture C, Vohl MC, Bouchard C, et al. (2015) Estimating genetic effect sizes under joint disease-endophenotype models in presence of gene-environment interactions. Front Genet 28: 248.

11. Barh D, Blum K, Madigan MA (2016) OMICS: biomedical perspectives and applications. CRC Press.

12. Hara K, Kadowaki T, Odawara M (2016) Genes associated with diabetes: potential for novel therapeutic targets? Expert Opin Ther Targets 20: 255-67.

13. Ferguson LR (2008) Dissecting the nutrigenomics, diabetes, and gastrointestinal disease interface: from risk assessment to health intervention. OMICS 12: $237-44$.

14. Lindgren CM, McCarthy MI (2008) Mechanisms of disease: genetic insights into the etiology of type 2 diabetes and obesity. Nat Clin Pract Endocrinol Metab 4: 156-63.

15. Hinney A, Vogel CI, Hebebrand J (2010) From monogenic to polygenic obesity: recent advances. Eur Child Adolesc Psychiatry 19: 297-310.

16. Desai M, Jellyman JK, Ross MG (2015) Epigenomics, gestational programming and risk of metabolic syndrome. Int J Obes (Lond) 39: 633-41.

17. Mead MN (2007) Nutrigenomics: The Genome-Food Interface. Environ Health Perspect 115: 582-9.

18. Elliott RM, Johnson IT (2007) Nutrigenomic approaches for obesity research. Obes Rev 8: 77-81.

19. Fenech M, El-Sohemy A, Cahill L, Ferguson LR, French TA, et al. (2011) Nutrigenetics and nutrigenomics: viewpoints on the current status and applications in nutrition research and practice. J Nutrigenet Nutrigenomics 4: 69-89.

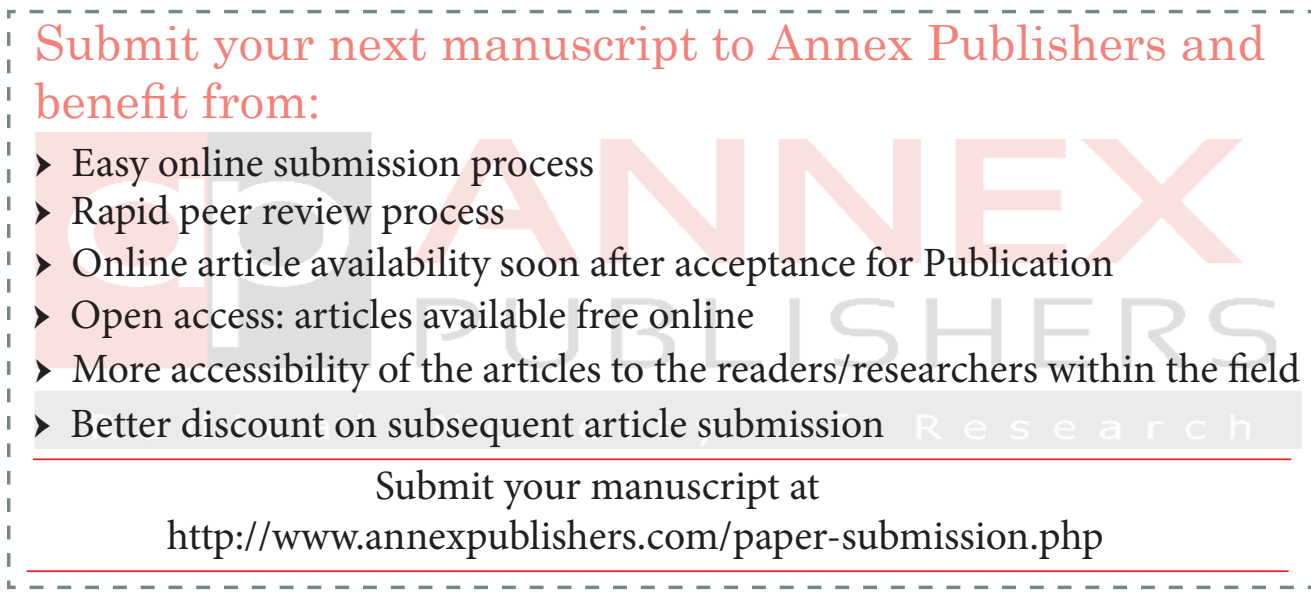

